\title{
Eficiência do atendimento do SUS nas microrregiões do estado de Santa Catarina ${ }^{1}$
}

\author{
Efficiency of care in the SUS microregions of Santa Catarina
}

\section{La eficiência de la atención del SUS en las microrregiones de Santa Catarina}

\author{
Leandro Politelo \\ Fundação Universidade Regional Blumenal \\ leandro_politelo@yahoo.com.br
}

\author{
Jorge Eduardo Scarpin \\ Universidade Federal do Paraná \\ jscarpin@gmail.com
}

\begin{abstract}
RESUMO
O objetivo deste estudo é avaliar a questão da eficiência do atendimento do SUS das vinte microrregiões do estado de Santa Catarina através da análise envoltória de dados (DEA). Esta pesquisa caracteriza-se como descritiva e com abordagem quantitativa. A amostra deste estudo é composta pelas 20 microrregiões de Santa Catarina. Os resultados desta pesquisa indicam que $65 \%$ das microrregiões pertencentes à amostra são eficientes. A média de eficiência do estado de Santa Catarina é de 97,04\%.75\% das microrregiões apresentam eficiência superior à média do estado e a microrregião menos eficiente apresentou escore de eficiência igual a 0,786, ou 78,6\%. Conclui-se que o SUS é eficiente quanto ao atendimento no estado de Santa Catarina.
\end{abstract}

Palavras-chave: Eficiência pública. Hospitais. Desempenho.

\begin{abstract}
The purpose of this study is to evaluate the question of efficiency of care SUS the twenty microregions of Santa Catarina through data envelopment analysis (DEA). This research is characterized as descriptive and quantitative approach. The sample consists of the 20 microregions of Santa Catarina. The results of this survey indicate that $65 \%$ of microregions belonging to the sample are efficient. The average efficiency of the state of Santa Catarina is $97.04 \% .75 \%$ of the microregions exhibit superior efficiency to the state average and less efficient microregion efficiency scores were equal to 0.786 , or $78.6 \%$. We conclude that the SUS is efficient as the service in the state of Santa Catarina.
\end{abstract}

Keywords: Public efficiency. Hospitals. Performance.

\section{RESUMEN}

El objetivo de este estudio es evaluar el tema de la eficiencia del tratamiento de veinte microrregiones de Santa Catarina a través de análisis envolvente de datos (DEA). Esta investigación se caracteriza como un enfoque descriptivo y cuantitativo. La muestra se compone de los 20 micro-regiones de Santa Catarina. Los resultados de este estudio indican que el $65 \%$ de las micro perteneciente a la muestra son eficientes. La eficiencia promedio del estado de Santa Catarina es de $97.04 \% .75 \%$ de la micro característica de la eficiencia superior a la media del estado y menos eficiente micro presentado la eficiencia puntuación igual a 0,786, o 78,6\%. Se concluye que el es eficiente ya que el servicio en el estado de Santa Catarina.

Palabras clave: Eficiencia públicas. Hospitales. Rendimiento.

\section{INTRODUÇÃO}

A eficiência dos hospitais e da prestação dos serviços de saúde como um todo tem sido muito discutido em um contexto geral (FONSECA; FERREIRA, 2009). Tal eficiência é

\footnotetext{
${ }^{1}$ Submetido em 14 de Janeiro de 2013. Aceito em 30 de Março de 2013. O artigo foi avaliado segundo o processo de duplo anonimato além de ser avaliado pelo editor. Editores responsáveis: Márcio Augusto Gonçalves e Lucas Maia dos Santos. Reprodução parcial ou total e trabalhos derivativos permitidos com a citação apropriada da fonte.
} 
facilmente compreendida e, até certo ponto, lógica, porém, as limitações presentes neste setor dificultam para que tal eficiência seja alcançada. Conforme Junqueira (1990) e Fonseca e Ferreira (2009), a saúde integra um dos direitos fundamentais do ser humano e por este motivo, Silva e Brandalize (2006) declaram que a qualidade da prestação de serviços de saúde deve ser uma constante preocupação em qualquer país.

Mills e Spencer (2005) afirmam que a qualidade na prestação de serviços pelos hospitais aos pacientes, que é o papel social destinado a estas organizações, deve ser algo intrínseco à sua atividade e, assim, não deve ser prestado com qualquer pretensão de qualidade e sem compromisso profissional. Junqueira (1990) também destaca que, por vezes, há criticas aos serviços de saúde, devido à falta de qualidade em sua prestação porque os funcionários não tratam os pacientes como clientes e focam apenas os seus interesses particulares.

Apesar de se entender a necessidade de os serviços da área de saúde serem prestados com qualidade, tem-se doutro lado, a limitação dos recursos destinados à saúde (PERIÓ; ARTELLS; MENEU, 2011) e o fato de o setor de saúde brasileiro ser um grande demandante de recursos públicos para prestação de seus serviços (SOUZA; NISHIJIMA; ROCHA, 2010).

Esta contradição entre a busca pela qualidade dos serviços de saúde e a limitação de recursos é discutida por diversos autores, como Marinho (2003), Mills e Spancer (2005) e Katharaki (2008). Neste ponto cabe destacar que uma coerente gestão de recursos faz-se oportuna (PAULY, 1987; MILLS; SPENCER, 2005) e até mesmo a obtenção de lucros ou superávits objetivando a perpetuidade da entidade, ainda que não seja o foco da organização (KEELER; MELNICK; ZWANZIGER, 1999; DENEFFE; MASSON, 2002).

Fonseca e Ferreira (2009) destacam que, já na década de 1990, havia, no Brasil, uma preocupação dos estudiosos na área da saúde e dos responsáveis do governo no que tange a qualidade e eficiência na prestação dos serviços de saúde no setor público. Conforme Staat (2006), esta também tem sido uma preocupação dos pesquisadores ao redor do mundo.

Pesquisadores indicam realidades diferentes quanto à eficiência do sistema de saúde. Al-Shammari (1999) considerou que os hospitais da Jordânia são eficientes, Staat (2006) destaca que os hospitais alemães apresentam eficiência média de 80\% e Chu (2011) conclui que os hospitais chineses apresentam significativa ineficiência. No Brasil, Cesconetto, Lapa e Calvo (2008) identificaram que apenas $21 \%$ dos hospitais catarinenses que analisaram eram eficientes, já Fonseca e Ferreira (2009) consideraram que os hospitais do estado de Minas Gerais são eficientes e Cunha (2011), ao analisar 70 hospitais filantrópicos brasileiros, identificou que apenas $14 \%$ destes eram eficientes.

A partir destes diferentes resultados perante a problemática apresentada, no que tange a eficiência na utilização dos recursos destinados à saúde, denota-se a questão objeto desta pesquisa: Qual o nível de eficiência do atendimento do Sistema Único de Saúde (SUS) nas microrregiões do estado de Santa Catarina? Deste modo o objetivo desta pesquisa é avaliar a questão da eficiência do atendimento do SUS das vinte microrregiões do estado de Santa Catarina através da análise envoltória de dados (DEA).

Este estudo se justifica na afirmação de Souza, Nishijima e Rocha (2010) de que, mesmo com o estabelecimento da descentralização na provisão e no financiamento das ações de saúde através do SUS dada pela Constituição Federal de 1988 com o objetivo de se obter maior eficiência no uso dos recursos, são poucos os estudos empíricos nacionais que avaliaram o desempenho dos municípios na gestão e uso destes recursos.

Sua relevância é vista pela necessidade de se identificar a eficiência na gestão dos recursos do SUS destinados a Santa Catarina perante os contrastes de ineficiências e eficiências apresentadas em pesquisas já realizadas que abordaram este tema, tendo em vista ainda a importância da qualidade destes serviços e que os recursos destinados a estes serviços são limitados (PERIÓ; ARTELLS; MENEU, 2011). 


\section{FUNDAMENTAÇÃO TEÓRICA}

Com o propósito de apresentar uma base teórica a fim de se atingir objetivo desta pesquisa aborda-se, na fundamentação teórica, temas relacionados à gestão e qualidade dos serviços hospitalares que aborda a importância da qualidade destes serviços à problemática perante a limitação de recursos destinados a estes serviços. Uma vez entendida a limitação de recursos aborda-se a importância da eficiência e do desempenho dos hospitais no uso dos recursos e, por fim, são relatados estudos relacionados ao tema desta pesquisa com objetivo de comparação dos resultados.

\subsection{GESTÃO E QUALIDADE DOS SERVIÇOS HOSPITALARES}

Os direitos fundamentais do ser humano, assegurados aos cidadãos brasileiros por meio da Constituição Federal do Brasil de 1988 compreendem, dentre outros, o direito à educação, à alimentação, à liberdade e à saúde (JUNQUEIRA, 1990; FONSECA; FERREIRA, 2009). Dentre estes direitos, esta pesquisa enfoca apenas aquele relacionado à saúde.

A questão da saúde brasileira, sem mister, denota-se aquém das necessidades dos cidadãos, e esta problemática advém de uma crise em sua própria estrutura e política de prestação de serviços (FONSECA; FERREIRA, 2009). Para os autores, esta crise aponta dois caminhos às políticas de saúde. O primeiro com a privatização dos serviços de saúde, que resultaria na inacessibilidade da maior parte da população à saúde, e o segundo, com a luta pela manutenção do direito conquistado na Constituição Federal de 1988, e a manutenção da garantia ao acesso universal e igualitário a serviços de qualidade.

Esta problemática é realidade não apenas no Brasil, mas em diversos países (CESCONETTO; LAPA; CALVO, 2008). Para os autores, o Brasil, não diferente de outros países, precisa estabelecer prioridades políticas para o controle de gastos públicos fronte a crescente demanda por recursos de saúde. Entende-se que a privatização dos serviços de saúde, por prejudicar grande parte da população, não solucionará os problemas relacionados à saúde pública e, a partir deste entendimento, deve-se adotar políticas alternativas de gestão.

O Brasil encontra-se, desta forma, fronte a duas questões contraditórias. Por um lado há a necessidade de se atender o direito do cidadão no que tange o acesso a serviços de saúde de qualidade e por outro, a limitação dos gastos públicos com saúde (MARINHO, 2003; MILLS; SPANCER, 2005; KATHARAKI, 2008). Neste cenário a eficiência do sistema de gestão pública é indispensável, questão já discutida em situação semelhante por Junqueira (1990).

A qualidade da prestação do serviço de saúde atenua esta problemática. Mills e Spencer (2005) salientam que o atendimento ao paciente, visto como papel social designado às organizações de saúde, não pode ser prestado com qualquer pretensão de qualidade, muito menos sem um compromisso profissional. Corroboram com esta assertiva Silva e Brandalize (2006) ao afirmarem que atendimento na área de saúde deve ser uma preocupação constante do estado e exige o comprometimento não só do estado, mas de todos os envolvidos na prestação do serviço.

Junqueira (1990) destaca também que a ausência de qualidade e o baixo desempenho dos serviços prestados na área de saúde, por vezes, são atrelados a falta de recursos, sejam humanos ou materiais, falta de qualificação profissional, porém, apenas dispor de recursos não garante a qualidade dos serviços, mas sim a aplicação eficientes de tais recursos. 
Tratando-se da questão das limitações dos gastos públicos em saúde, a geração contínua de recursos, o controle de custos e a obtenção lucros/superávits devem compor os objetivos das organizações de saúde (PAULY, 1987; MILLS; SPENCER, 2005). Ainda que este último não seja o foco da organização (KEELER; MELNICK; ZWANZIGER, 1999; DENEFFE; MASSON, 2002), deve ser esperado, uma vez que a má gestão pode levar a ausência de recursos e, consequentemente, à "morte" da entidade.

Apesar da limitação de recurso, há casos em que a organização não deve pesar a relação entre custos e o atendimento, já que a preservação da vida é primordial e objetivo maior de qualquer organização de saúde, porém, a gestão dos custos e a geração de recursos são essenciais para a manutenção dos fluxos de caixa da entidade e, por conseguinte, para a sua perpetuidade (MORRISEY; WEDIG; HASSAN, 1996; MILLS; SPENCER, 2005).

Em suma, a ênfase no aspecto social atrelado aos hospitais já destacada por Reder em 1965, tendo em vista o atendimento ao maior número possível de pacientes, confronta-se com a limitação do orçamento destinado ao sistema público de saúde corroborando com a dicotomia de gestão de custos e limitação de recursos fronte a qualidade e amplitude dos serviços prestados.

Esta contradição de objetivos tem fomentado movimentos internacionais, dentre os quais se destaca a Reforma do Estado que contribuiu para a proliferação de experiências na área da administração pública cujas características básicas incluíam maior flexibilidade gerencial no que tange à compra de insumos e materiais, gestão de recursos financeiros, contratações e dispensa de recursos humanos, e a implantação de gestão com foco na qualidade dos serviços prestados e satisfação dos usuários (IBAÑES; VECINA NETO, 2007).

Diante desta problemática na qual a política governamental brasileira está inserida e, mediante a influência dos movimentos internacionais com relação à Reforma do Estado, Ibañes e Vecina Neto (2007) salientam que a intenção de "enxugar" o tamanho do Estado sempre esteve presente, e seu início se daria com a transferência de serviços, como saúde, educação e cultura, para entidade privadas por serem estes serviços considerados como não exclusivos do Estado, porém, tal atitude traria à realidade a marginalização de grande parte da população (FONSECA; FERREIRA, 2009).

Ferlie et al. (1999), em sua análise quanto às tendências de reforma do Estado no contexto internacional, caracterizam quatro modelos da nova administração pública: 1) o modelo do impulso para a eficiência; 2) modelo de maior controle financeiro; 3) modelo downsizing e descentralização e; 4) modelo da busca pela excelência, baseado na Escola de Relações Humanas, com ênfase na "cultura organizacional".

O primeiro modelo apresentado por Ferlie et al. (1999) versa sobre a aproximação entre os serviços públicos e os serviços privados e preconiza a transformação do setor público a partir da introdução de padrões de eficiência desenvolvidos pelo setor privado. O segundo modelo, ou modelo de maior controle financeiro aborda a introdução de sistemas de custos e auditorias aos aspectos financeiros e profissionais, com o estabelecimento de objetivos claros, monitoramento do desempenho organizacional e da desregulamentação do mercado de trabalho.

O terceiro modelo proposto por Ferlie et al. (1999) é o modelo downsizing e descentralização cujo propósito baseia-se na separação entre o financiamento público e a dotação do setor autônomo, ou privado, com a substituição da gestão hierárquica pela a gestão por contrato, constituição de organizações separadas para a compra e para a prestação de serviços e a redução significativa da folha de pagamento das instituições públicas.

O quarto modelo, ou modelo da busca pela Excelência, baseado na Escola de Relações Humanas, com ênfase na "cultura organizacional" é o modelo de orientação para o serviço público. Para os Ferlie et al. (1999) este é o modelo menos aplicado, e sua origem dá-se pela fusão entre os modelos definidos pelos serviços públicos e privados, conferindo ao modelo 
aspectos administrativos qualitativos presente nos serviços privados aos serviços públicos, incluindo a responsabilidade dos serviços com os usuários e os cidadãos, deixando de considerá-los como meros consumidores ou simples usuários.

Nos modelos propostos por Ferlie et al. (1999) é perceptível a busca pela introdução, ao menos parcial, do setor privado nos serviços cuja obrigação compete ao Estado ou então, de práticas comumente realizadas pelo setor privado para controle e eficácia da gestão. Ibañes e Vecina Neto (2007) destacam que as buscas alternativas para a gestão em saúde, principalmente de hospitais, objetivam a eficiência dos serviços públicos de saúde. Vendo os hospitais como agências complexas, os autores concluem que a construção de modelos que aperfeiçoem a capacidade de produção de resultados sanitários não se enquadra na busca de maior competitividade, mas na busca de uma agência mais efetiva para a sociedade.

Não há de se negar a presença da função social nos hospitais quando os serviços por eles prestados envolvem um dos direitos fundamentais do ser humano. Também há de se admitir que os recursos disponíveis à área de saúde no Brasil são limitados e que a qualidade na prestação do serviço só é possível a partir de uma gestão eficaz destes recursos. A redução do Estado com atuação exclusiva do setor privado na saúde não pode ser vista como solução plena para os problemas atrelados à saúde, assim, a presença do Estado faz-se necessária e, perante a latente questão de limitação de recursos e qualidade na prestação de serviços, a eficácia hospitalar mostra-se como essencial e indispensável.

\subsection{EFICIÊNCIA E DESEMPENHO DE HOSPITAIS}

A busca pela melhoria da eficiência na prestação de serviços relacionados à saúde, com o desenvolvimento de novas formas de atendimento da população provém do processo de descentralização das ações do estado cujo foco é a eficiência destes serviços, compreendendo também o princípio de universalização do acesso à saúde a fim de impactar positivamente a qualidade de vida da população (FONSECA; FERREIRA, 2009).

Eficiência pode ser entendida como o consumo mínimo de entradas para um determinado nível de saídas ou, como o aumento das saídas mantendo-se um determinado nível de entradas (CHUANG; CHANG; LIN, 2011), neste sentido, eficiência na prestação de serviços em saúde é a relação entre o custo e o impacto destes na saúde da população, mantendo um nível de qualidade determinado (VIACAVA et al., 2012), ou seja, proporcionar qualidade e benefícios com o menor custo ou, com um mesmo custo, proporcionar aumento na qualidade e benefícios aos usuários dos serviços de saúde.

Em geral entende-se eficiência como a capacidade, ou habilidade, do uso adequado dos recursos disponíveis com o objetivo de se alcançar o resultado pretendido (FONSECA; FERREIRA, 2009). Assim, "a eficiência pode ser considerada uma medida da capacidade que agentes ou mecanismos têm para melhor atingir seus objetivos, produzindo o efeito por eles esperado, em função dos recursos disponíveis". (FONSECA; FERREIRA, 2009, p. 204). A eficiência na dinâmica da prestação de serviços de saúde pode ser verificada na figura 1.

Figura1 - Matriz das dimensões da avaliação do sistema de saúde proposta pelo PRO-ADESS

\begin{tabular}{|c|c|}
\hline \multicolumn{2}{|c|}{ CONDIÇÕES DE SAÚDE DA POPULAÇÃO } \\
\hline$\underline{\text { Morbidade }}$ & $\underline{\text { Estado funcional }}$ \\
\hline $\begin{array}{l}\text { Ocorrência de } \\
\text { sintomas, doenças, } \\
\text { traumas e deficiências }\end{array}$ & $\begin{array}{l}\text { Ocorrência de limitação } \\
\text { ou restrição na realização } \\
\text { de atividades cotidianas } \\
\text { típicas }\end{array}$ \\
\hline$\underline{\text { Bem-estar }}$ & $\underline{\text { Mortalidade }}$ \\
\hline
\end{tabular}




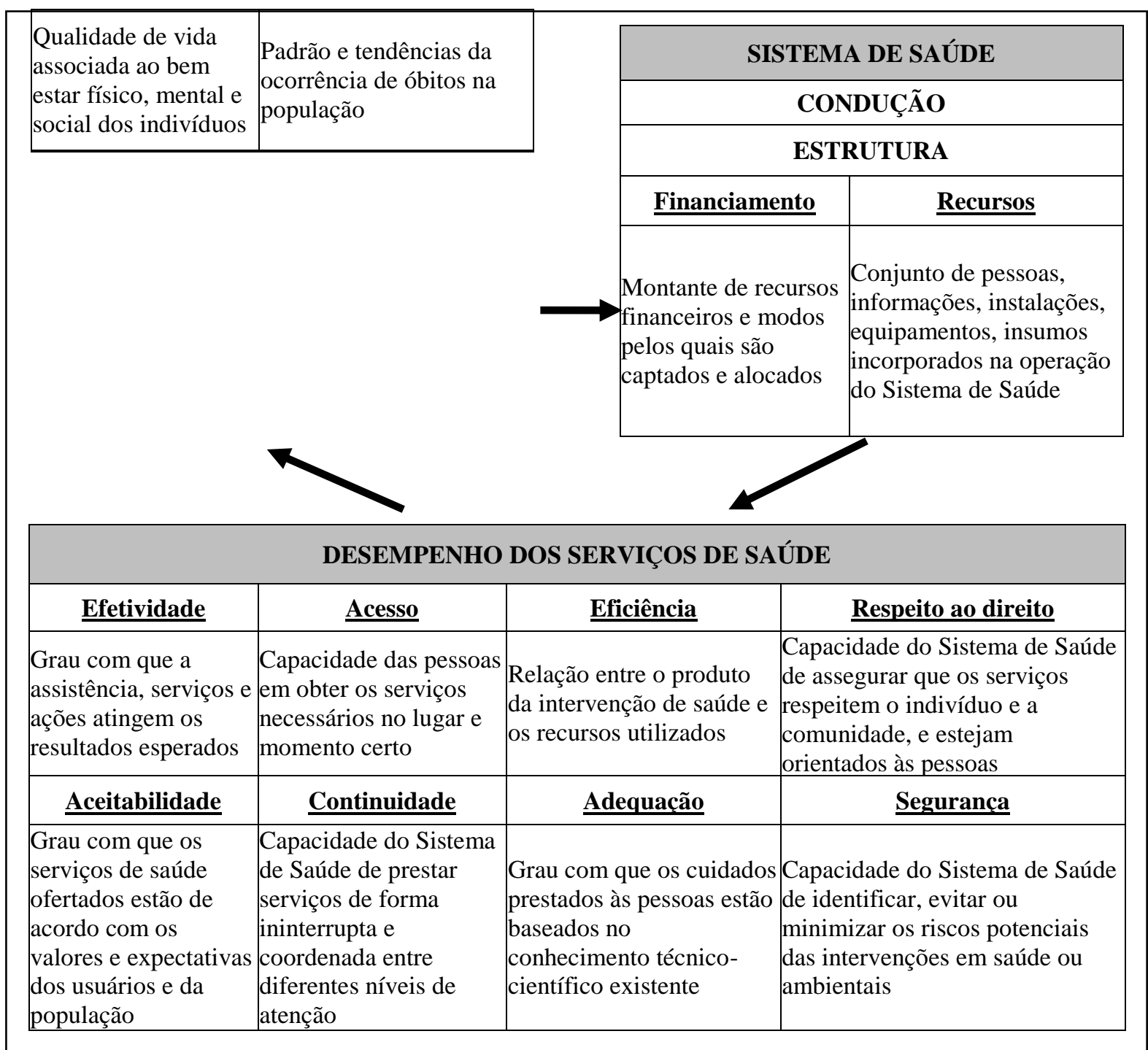

Fonte: Politelo et al. (2012, p. 5)

Na figura 1 fica evidente a relação entre eficiência na prestação de serviços de saúde e a qualidade de vida da sociedade. O desempenho dos serviços de saúde, que compreendem a eficiência, efetividade, segurança, continuidade, adequação, entre outros, como aceitabilidade e acesso dependem diretamente dos recursos e financiamentos do sistema de saúde, que por sua vez são alimentados pela sociedade.

Como identificado na figura 1 , o desempenho dos serviços de saúde interferem diretamente nas condições de saúde da população, de modo que, serviços de maior qualidade impactam em melhores condições de saúde. Assim, O desempenho dos serviços prestados por entidade de saúde impacta no volume de recursos que irá receber para financiamento de suas atividades bem como proporcionar melhor qualidade de vida à comunidade que atende.

Mesmo que haja esta relação, o financiamento dos serviços de saúde é limitado, de modo que o uso eficiente destes recursos mostra-se como importante objeto de estudos (MILLS; SPANCER, 2005). A eficiência na utilização dos recursos recebe maior destaque quando aceita-se que a qualidade destes serviços é primordial, a ponto de superar, em certos casos, a importância da gestão de custos (MORRISEY; WEDIG; HASSAN, 1996; MILLS; SPENCER, 2005).

Conforme Chuang, Chang e Lin (2011), quando um hospital assume a responsabilidade social de oferecer cuidado e tratamento médico ao público, sua eficiência 
deve ser direcionada a minimização dos gastos sem comprometer a qualidade. De igual forma Barnum et al. (2011) destacam a importância da eficiência da saúde em virtude do crescente aumento dos custos de saúde e do número de pessoas atendidas pelos programas públicos de saúde.

Deve-se considerar também que a estrutura do sistema de saúde interfere no desempenho da prestação de serviços relacionados à saúde, assim, sua estruturação e funcionamento, geralmente decorrentes de determinantes sociais, econômicos e políticos, devem ser capazes de garantir o atendimento das necessidades de saúde (VIACAVA et al., 2012). Apesar da evidente necessidade de eficiência na utilização dos recursos destinados à saúde, as pesquisas realizadas nesta área apresentam uma realidade contrária, ou seja, utilização não eficientes dos recursos disponíveis. Como exemplo cita-se as pesquisas de Sarkis; Talluri (2002), Staat (2006) e Cesconetto, Lapa e Calvo (2008).

Dentro da realidade brasileira, estudos têm apontado para a fragilidade do SUS (Sistema Único de Saúde), indicando que sua eficiência não envolve apenas aspectos relacionados à restrição de recursos e problemas de financiamento, propondo o aumento de sua eficiência a partir de métodos adequados de medição, gerenciamento e controle dos serviços prestados juntamente com o estabelecimento de melhores políticas públicas. (GUERRA; SOUZA; MOREIRA, 2012).

Em geral, a qualidade nos serviços de saúde interfere diretamente na qualidade de vida da sociedade, assim, a busca pela maximização no uso de recursos destinados à saúde tendo em vista a qualidade na prestação de serviços deve ser constante (SILVA; BRANDALIZE, 2006). Isto posto, a análise de sua eficiência é importante ou, até mesmo, necessária.

\subsection{Trabalhos correlatos}

Neste tópico é apresentada uma síntese de estudos realizados no âmbito nacional e internacional relacionados à eficiência dos serviços de saúde.

Al-Shammari (1999), teve como objetivo medir e avaliar a eficiência dos cuidados de saúde dos hospitais utilizando a Análise Envoltória de Dados (DEA). Com base em dados de três anos (1991 a 1993) de 15 hospitais da Jordânia o autor concluiu que eles são relativamente eficientes, mas que há casos em que os hospitais podem melhorar o número de atendimento aos usuários, otimizando os recursos disponíveis.

Em um cenário de pesquisas com resultados controversos, Staat (2006) pesquisou dois segmentos de hospitais alemães que compreendeu uma amostra de 1700 hospitais localizados nos antigos estados federais da Alemanha, todos com um departamento de cirurgia. $\mathrm{O}$ autor concluiu que a eficiência dos hospitais alemães é de aproximadamente 80\%. Staat (2006) Identificou também que o segmento I de hospitais era menos eficiente que o segmento II, apontando que os esforços de melhorias no sistema de saúde deveriam focar o segmento I.

Gonçalvez (2008) buscou compreender o impacto no setor hospitalar, em termos de eficiência, resultante da introdução de um novo modelo de gestão nos hospitais portugueses. Como resultado, Gonçalvez (2008) identificou que no período por ele analisado, os hospitais S.A. (Sociedade Anônima) formados a partir do novo modelo de gestão foram menos eficientes do que os hospitais SPA (hospitais tradicionais que mantiveram o estatuto de entidades do Setor Público Administrativo). Identificou também que a fronteira de eficiência melhorou no triênio 2002 a 2004 em virtude do clima competitivo.

Cesconetto, Lapa e Calvo (2008), a partir de dados de 2003, avaliaram a eficiência produtiva de 112 hospitais conveniados ao SUS no estado de Santa Catarina a fim de atender ao objetivo proposto de verificar quais hospitais eram eficientes quanto ao uso dos recursos disponíveis. Como resultado, verificaram que apenas 23 dos 112 hospitais eram eficientes. Identificaram que a eficiência dos hospitais do estado seria alcançada com o aumento de $15 \%$ 
no número de internações ou com a redução em $25 \%$ no número de profissionais da área, $17 \%$ do número de leitos e de $13 \%$ no valor de AIH (Autorização de Internação Hospitalar).

Fonseca e Ferreira (2009), ao realizarem sua pesquisa, objetivaram analisar os níveis de eficiência em unidades institucionais homogêneas do setor de saúde das microrregiões de Minas Gerais e, a partir dos resultados da pesquisa, concluem que os hospitais das microrregiões do estado de Minas Gerais são eficientes por apresentarem índices de eficiência superiores a média do estado. Apesar de declararem a eficiência das microrregiões, os autores identificaram que há uma considerável disparidade entre as microrregiões quanto à gestão dos recursos destinados à saúde.

Com objetivo semelhante a Fonseca e Ferreira (2009), utilizando uma amostra de 366 municípios de São Paulo, Souza, Nishijima e Rocha (2010) avaliaram o grau de eficiência do setor público hospitalar utilizando dados dos anos de 1998 e 2003. Os resultados da pesquisa de Souza, Nishijima e Rocha (2010) indicam que os municípios com maior população são os mais ineficientes. Os autores identificaram também que os municípios mais eficientes são os que contratam um maior número de leitos de hospitais privados e de leitos universitários, que possuem menor população, maior número de internações e menor tempo médio de internações.

Em virtude de implementações de reformas dos cuidados de saúde na China, Chu (2011) buscou evidenciar empiricamente a ineficiência dos hospitais chineses utilizando a Análise Envoltória de Dados. A partir de dados dos anos de 2004 a 2008 de 463 hospitais, Chu (2011) identificou significativa ineficiência dos hospitais no período analisado. Conforme o autor, neste período houve um crescimento de produtividade no setor em virtude da mudança tecnológica, refletindo no aumento de receitas com prescrição em excesso de medicamentos, cuidados desnecessários e utilização de tratamentos de alta tecnologia fato que permitiu ao autor, em conjunto com os resultados de sua pesquisa, concluir que o desenvolvimento econômico não implica, necessariamente, em um melhor desempenho e maior eficácia dos hospitais.

Cunha (2011) apresentou como objetivo de sua pesquisa, desenvolver um modelo organizacional para avaliação de desempenho para os hospitais filantrópicos e, partindo deste modelo, comparar a eficiência das organizações da amostra. A partir de respostas obtidas de 70 hospitais o autor considerou 10 hospitais como eficientes, sendo a maioria destes pertencentes a região Sul e Sudeste e ao estado de Minas Gerais. O autor conclui que o Ministério da Saúde deve atuar com maior ênfase a fim de que os hospitais filantrópicos recebam subvenções em tempo hábil de modo que possam ser mais eficientes.

Utilizando uma amostra de 98 províncias italianas, Nicola, Gitto e Mancuso (2012) buscaram identificar o nível de eficiência dos hospitais italianos e quais variáveis de ambiente afetam sua eficiência. Utilizando a Análise Envoltória de Dados e análise CART, os autores descrevem que a variável mais influente na eficiência dos hospitais é o percentual de leitos gerenciados diretamente pelo governo regional. Assim, os autores concluem que uma melhor administração dos leitos, pelo governo, pode aumentar a eficiência das organizações de saúde, mas destacam que, nesta gestão, deve-se atentar para a mobilidade dos doentes.

Estes estudos realizados com objetivo de analisar a eficiência do sistema de saúde, apesar de não utilizar variáveis idênticas, indicam realidades diferentes nos países e mesmo entre as regiões brasileiras. Esta situação de coexistência de eficiência e ineficiências já foi destaca no estudo de Viacava et al. (2012). Neste aspecto, faz-se oportuno identificar a eficiência na utilização de recursos destinados à saúde em Santa Catarina.

\section{METODOLOGIA}

Para avaliar a eficiência do atendimento do SUS nas microrregiões do estado de Santa Catarina realizou-se uma pesquisa descritiva, com abordagem quantitativa, por meio de 
pesquisa documental. A característica descritiva é vista quando da exposição da eficiência das microrregiões analisadas, apresentando, assim, descrição da amostra analisada (VERGARA, 2000).

No tocante a abordagem do problema, conforme Richardson (1999), a pesquisa é classificada como quantitativa, devido ao uso de instrumentos estatísticos nas fases de coleta, tratamento e análise dos dados, no caso, uso de estatística descritiva para caracterizar a amostra da pesquisa e apresentar informações gerais quanto as variáveis utilizadas e aplicação da Análise Envoltória de Dados (DEA) para identificar a eficiência das microrregiões de Santa Catarina quanto ao uso dos recursos destinados à saúde.

Em relação ao procedimento, classifica-se como pesquisa documental por utilizar-se de informações do ano de 2009 presentes nos relatórios públicos divulgados pela secretaria da Saúde no sítio do DATASUS.

\subsection{População e amostra}

A população estudada é composta por 20 microrregiões do estado de Santa Catarina. A classificação das microrregiões de Santa Catarina bem como os municípios pertencentes às microrregiões baseou-se na classificação efetuada pelo DATASUS. Todas as microrregiões foram consideradas para a análise de modo que a amostra da pesquisa perfaz, de igual modo, as 20 microrregiões do estado. Em virtude de os dados das microrregiões serem compostos a partir dos dados dos municípios, aqueles que não apresentavam todos os dados necessários não foram considerados para a pesquisa. As microrregiões com sua respectiva quantidade de municípios e a quantidade de municípios cujos dados foram utilizados são apresentados na tabela 1.

Tabela 1 - População e amostra da pesquisa

\begin{tabular}{l|c|c}
\hline \multicolumn{1}{c|}{ Microrregiões } & Total de municípios & Municípios considerados para a amostra \\
\hline Florianópolis & 9 & 4 \\
Tabuleiro & 5 & 3 \\
Tijucas & 7 & 5 \\
Canoinhas & 12 & 10 \\
Joinville & 11 & 4 \\
São Bento do Sul & 3 & 3 \\
Chapecó & 38 & 15 \\
Concórdia & 15 & 10 \\
Joaçaba & 27 & 17 \\
São Miguel do Oeste & 21 & 13 \\
Xanxerê & 17 & 7 \\
Campos de Lages & 18 & 11 \\
Curitibanos & 12 & 5 \\
Araranguá & 15 & 7 \\
Criciúma & 10 & 6 \\
Tubarão & 19 & 10 \\
Blumenau & 15 & 9 \\
Itajaí & 12 & 6 \\
Ituporanga & 7 & 5 \\
Rio do Sul & 20 & 13 \\
\hline Total & 293 & 163 \\
\hline Font: Dados Pesquisa & & \\
\hline
\end{tabular}

Fonte: Dados da Pesquisa.

Dos 293 municípios do estado de Santa Catarina, 55,63\% deles apresentavam todos os dados necessários para a realização desta pesquisa. A microrregião de Joinville é a 
microrregião com maior número de municípios com dados desatualizados. Apenas para a microrregião de São Bento do Sul foi possível utilizar os dados de todas as cidades. Em geral, puderam-se utilizar todas as microrregiões para a análise visto que todas apresentaram dados válidos para a pesquisa.

\subsection{Coleta e análise dos resultados}

Os dados dos municípios foram coletados a partir do sitio oficial do DATASUS cuja fonte é o Ministério da Saúde. Os dados do Ministério da Saúde são produzidos através do Cadastro Nacional dos Estabelecimentos de Saúde no Brasil, Sistema de Informações sobre Mortalidade e Sistema de Informações Ambulatoriais, que divulgam indicadores de recursos, produção e resultados na área da saúde, classificados por microrregiões. Desta forma, os dados para realização do estudo são classificados como dados secundários.

O período de referência utilizado foi o ano de 2009 por ser o período mais recente com disponibilidade completa das informações. Os dados foram coletados a partir de diferentes tabelas da base de dados da pesquisa. Para o cálculo das medidas de eficiência, foram consideradas três variáveis relacionadas aos produtos e três relacionadas aos insumos.

As variáveis que medem a produção referem-se às internações per capita, total de procedimentos ambulatoriais per capita e o inverso da taxa de mortalidade. Já as variáveis que contêm os insumos utilizados referem-se à capacidade ambulatorial, o valor médio das internações e a quantidade de leitos por hospitais. No quadro 1 apresentam-se a descrição das variáveis utilizadas bem como a referência de pesquisas anteriores nas quais tais variáveis foram utilizadas.

Quadro 1 - Variáveis utilizadas no estudo.

\begin{tabular}{|c|c|c|c|}
\hline Dados & Variável & Descrição & Autores \\
\hline \multirow[b]{3}{*}{ Insumos } & $\begin{array}{l}\text { Capacidade } \\
\text { ambulatorial. }\end{array}$ & $\begin{array}{l}\text { Número de equipamentos existentes em } \\
\text { dezembro de } 2009 .\end{array}$ & Fonseca e Ferreira (2009). \\
\hline & $\begin{array}{l}\text { Valor médio } \\
\text { das internações. }\end{array}$ & $\begin{array}{l}\text { Valor médio de internação no ano de } \\
2009 .\end{array}$ & Cunha (2011). \\
\hline & $\begin{array}{l}\text { Quantidade de } \\
\text { leitos por } \\
\text { hospitais. }\end{array}$ & $\begin{array}{l}\text { Número de leitos hospitalares em } \\
\text { dezembro de } 2009 \text {. }\end{array}$ & $\begin{array}{l}\text { Staat (2006); } \\
\text { Santos, Carvalho e Lírio (2008); } \\
\text { Cesconetto, Lapa e Calvo (2008); } \\
\text { Souza, Nishijima e Rocha (2010); } \\
\text { Cunha (2011). }\end{array}$ \\
\hline \multirow{3}{*}{ Produto } & $\begin{array}{l}\text { Internações per } \\
\text { capita. }\end{array}$ & $\begin{array}{l}\text { Número total de internações no ano de } \\
2009 \text { dividido pelo número de } \\
\text { população. }\end{array}$ & Cunha (2011). \\
\hline & $\begin{array}{l}\text { Total de } \\
\text { procedimentos } \\
\text { ambulatoriais } \\
\text { per capita. }\end{array}$ & $\begin{array}{l}\text { Quantidade de procedimentos aprovados } \\
\text { para pagamento pelas Secretarias de } \\
\text { Saúde no ano de } 2009 \text { dividido pelo } \\
\text { número de população. }\end{array}$ & Fonseca e Ferreira (2009). \\
\hline & $\begin{array}{l}\text { Inverso da taxa } \\
\text { de mortalidade. }\end{array}$ & $\begin{array}{l}\text { Taxa inversa de mortalidade da } \\
\text { organização de saúde no ano de } 2009 .\end{array}$ & Santos, Carvalho e Lírio (2008). \\
\hline
\end{tabular}

Fonte: Dados da Pesquisa.

Como o estudo é baseado nos informações das microrregiões e os dados obtidos referem-se aos municípios, procedeu-se da seguinte forma para compilação das informações das microrregiões: a) Para a variável "Capacidade ambulatorial" efetuou-se a soma de cada município; b) Calculou-se a média do valor médio das internações dos municípios para se obter o "Valor médio das internações"; c) Para a variável "Quantidade de leitos por hospitais" somou-se a quantidade de leitos e o número de leitos dos municípios e destes totais realizouse a divisão da quantidade de leitos pelo número total de hospitais; d) A partir da soma das internações divida pela soma da população das cidades obteve-se a variável "Internações per 
capita"; e) A variável "Total de procedimentos ambulatoriais per capita" foi calculada a partir da soma dos procedimentos ambulatoriais dividido pela soma da população das cidades; f) Para a variável "Inverso da taxa de mortalidade", dividiu-se o número 1 pela média da taxa de mortalidade das cidades de cada microrregião.

Utilizou-se o método não paramétrico de análise envoltória de dados (DEA), que utiliza programação linear para estimativa da fronteira de eficiência, para análise dos dados. $\mathrm{O}$ DEA é indicado para este tipo de análise porque é capaz de incorporar diversos insumos (inputs) e produtos (outputs) para calcular a eficiência das unidades tomadoras de decisão, designada de DMUs (GOMES; MANGABEIRA, 2004). Para tal, utilizou-se o software MaxDEA.

O método de análise envoltória de dados (DEA) utiliza a programação matemática para obter a eficiência relativa dos resultados obtidos por gestores com base em fatos passados (BANKER; CHARNES; COOPER, 1984). Um indicador que varia de 0 a 1 é fornecido pelo DEA e, quanto mais próximo a 1, mais eficiente, assim, escores igual a 1 indicam eficiência (MACEDO; BARBOSA; CAVALCANTE, 2009).

Existem dois modelos do DEA que são os mais utilizados, o CCR (CHARNES; COOPER; RHODES, 1978) e o BCC (BANKER; CHARNES; COOPER, 1984). Também conhecido como Constant Returns to Scale (CRS) o modelo CCR avalia a eficiência total, determinando as DMUs de eficiência e qual a distância existente entre a fronteira de eficiência e as DMUs não eficientes. Já o modelo BCC, também conhecido como Variable Returns to Scale (VRS) "[...] permite a projeção de cada DMU ineficiente sobre a superfície de fronteira (envoltória) determinada pelas DMUs eficientes de tamanho compatível" (MACEDO; BARBOSA; CAVALCANTE, 2009, p. 68).

Existe a necessidade de fixação da ótica de análise, além da escolha do modelo, se orientada pelos insumos ou pelos produtos. A orientação pelos insumos visa minimizar proporcionalmente as quantidades de insumos, mantendo fixas as quantidades de produtos, neste, o objetivo é reduzir insumos e manter uma mesma produção. Já o orientado pelos produtos, visa maximizar proporcionalmente as quantidades de produtos, mantendo as quantidades de insumos fixas, ou seja, com uma determinada quantidade de insumos, obter a máxima produção (FERREIRA; GOMES, 2009). Na aplicação do DEA neste estudo, utilizase o modelo BCC com orientação voltada para a maximização dos produtos, entendendo que, na situação atual da saúde pública, com as limitações de recursos presentes, deve-se otimizar o uso dos recursos disponíveis.

\section{DESCRIÇÃO E ANÁLISE DOS RESULTADOS}

A estatística descritiva das variáveis utilizadas na pesquisa para cálculo da eficiência do SUS em Santa Catarina é apresentada na tabela 2.

Tabela 2 - Estatística descritiva das variáveis

\begin{tabular}{l|c|c|c|c|c|c}
\hline \multicolumn{1}{c|}{ Variáveis } & n & Mínimo & Máximo & Média & Mediana & $\begin{array}{c}\text { Desvio- } \\
\text { padrão }\end{array}$ \\
\hline Capacidade ambulatorial & 20 & 38,00 & 873,00 & 315,55 & 299,50 & 208,45 \\
Valor médio das internações & 20 & 496,26 & $1.576,25$ & 670,23 & 596,26 & 255,45 \\
Leitos por hospitais & 20 & 1,65 & 9,50 & 3,07 & 2,68 & 1,61 \\
Internações per capita & 20 & 0,05 & 0,09 & 0,08 & 0,08 & 0,01 \\
Total de procedimentos ambulatoriais per & & & & & & \\
capita & 20 & 8,75 & 61,36 & 17,99 & 13,69 & 13,99 \\
Inverso da taxa de mortalidade & 20 & 0,23 & 0,69 & 0,44 & 0,44 & 0,12 \\
\hline
\end{tabular}

Fonte: Dados da Pesquisa. 
Dentre as variáveis consideradas inputs, a que apresentou maior desvio-padrão, ou seja, a variável com maior discrepância entre o valor mínimo e máximo, conforme a tabela 2 , foi a variável "valor médio das internações", esta constatação indica uma grande diferenciação nos custos de internação nas microrregiões catarinenses. Percebe-se que o valor máximo é aproximadamente 4 vezes o valor mínimo do custo médio de internações. A variável "leitos por hospitais" apresentou o menor desvio-padrão entre os inputs, indicando uma maior uniformidade quanto à quantidade de leitos presente nos hospitais das microrregiões de Santa Catarina.

Conforme a tabela 2, a variável com maior desvio-padrão, dentre aquelas variáveis consideradas outputs, é a variável "total de procedimentos ambulatoriais per capita" que pode estar relacionada às diferentes capacidades ambulatoriais, já que esta variável (inputs) apresenta variação considerável entre seu valor mínimo e máximo. Destaca-se que as variações dos outputs são pequenas, já as variações dos inputs são grandes (exceto para a variável "leitos por hospitais"). Estas variações dos inputs podem estar relacionadas às variações populacionais entre as microrregiões, tal assertiva se apoia na questão de que os inputs normalizados pela população (per capita) possuem baixo desvio-padrão. Deste modo considera-se que os dados são válidos para a presente análise.

A tabela 3 apresenta a análise de eficiência dos hospitais das microrregiões de Santa Catarina com atendimento pelo SUS, realizada através do cálculo do DEA.

Tabela 3 - Score de eficiência das microrregiões de Santa Catarina

\begin{tabular}{l|c}
\hline \multicolumn{1}{c|}{ Microrregiões (DMUs) } & Score de Eficiência \\
\hline Araranguá & 1 \\
\hline Blumenau & 1 \\
\hline Campos de Lages & 1 \\
\hline Chapecó & 1 \\
\hline Concórdia & 1 \\
\hline Curitibanos & 1 \\
\hline Florianópolis & 1 \\
\hline Ituporanga & 1 \\
\hline São Bento do Sul & 1 \\
\hline São Miguel do Oeste & 1 \\
\hline Tabuleiro & 1 \\
\hline Tijucas & 1 \\
\hline Xanxerê & 1 \\
\hline Rio do Sul & 0,981 \\
\hline Tubarão & 0,972 \\
\hline Itajaí & 0,954 \\
\hline Criciúma & 0,941 \\
\hline Joaçaba & 0,893 \\
\hline Canoinhas & 0,881 \\
\hline Joinville & 0,786 \\
\hline Fonte: Dados & 1 \\
\hline
\end{tabular}

Fonte: Dados da Pesquisa.

A tabela 3 permite observar que 13 das 20 microrregiões catarinenses apresentaram eficiência máxima quanto aos atendimentos realizados pelo SUS, são elas: Araranguá, Blumenau, Campos de Lages, Chapecó, Concórdia, Curitibanos, Florianópolis, Ituporanga, São Bento do Sul, São Miguel do Oeste, Tabuleiro, Tijucas e Xanxerê. Dado a orientação do DEA para os produtos, estes resultados indicam que estas cidades obtiveram o maior número de atendimentos (outputs) mantendo a quantidade de recursos disponíveis (inputs).

No que tange ao uso dos recursos de saúde disponíveis aos hospitais com atendimento pelo SUS, 65\% da amostra pesquisada mostrou-se eficiente. Este resultado difere-se dos 
resultados do estudo de Cesconetto, Lapa e Calvo (2008) e de Cunha (2011), que evidenciaram uma minoria de suas amostras como eficientes. Destaca-se que a pesquisa de Cesconetto, Lapa e Calvo (2008) também teve como foco o estado de Santa Catarina, assim, evidencia-se uma melhoria quanto à gestão dos recursos destinados à saúde em Santa Catarina no período de 2003 (base da pesquisa de Cesconetto, Lapa e Calvo) a 2009 (período base desta pesquisa).

Das 13 microrregiões eficientes, destaca-se a microrregião de Florianópolis, que apresentou os maiores valores de inputs em capacidade ambulatorial e leitos por hospital e a sungo maior valor para o valor médio das internações e obteve resultado de eficiência quanto ao uso de seus recursos. A partir dos resultados de eficiência das microrregiões, a média de eficiência de Santa Catarina, quanto ao atendimento do SUS, é de 0,9704, ou 97,04\% o que denota uma considerável eficiência do estado, mas que pode aumentar, em média, sua produção em $2,86 \%$ para atingir a eficiência plena.

Os resultados da pesquisa indicam um significativo índice de eficiência das microrregiões pesquisadas, dado que a eficiência média é de aproximadamente $97 \%$. Este resultado permite afirmar que as microrregiões catarinenses são eficientes, uma vez que $75 \%$ das microrregiões pesquisadas possuem eficiência superior à média do estado, e as demais microrregiões ainda possuem um nível de eficiência alto.

Comparando os resultados à pesquisa de Fonseca e Ferreira (2009) pode-se concluir que as microrregiões e, consequentemente o estado de Santa Catarina, possui eficiência superior ao estado de Minas Gerais, uma vez que a eficiência média deste estado é $78 \%$ e daquele, de 97,04\%. Ainda, apenas $18 \%$ das microrregiões mineiras apresentaram nível máximo de eficiência em contraponto a $65 \%$ das microrregiões catarinenses.

Em comparação ao cenário internacional, a realidade quanto à eficiência catarinense se contrapõe a significativa ineficiência dos hospitais chineses apontada por Chu (2011). O destaque quanto ao alto nível de eficiência média catarinense assemelha-se a eficiência dos hospitais da Jordânia (AL-SHAMMARI, 1999) e, de comparada à eficiência dos hospitais alemães (STAAT, 2006), percebe-se uma eficiência superior dos hospitais catarinense.

As 7 microrregiões não eficientes são: Rio do Sul, Tubarão, Itajaí, Criciúma, Joaçaba, Canoinhas e Joinville. Destas destaca-se a microrregião de Joinville como a menos eficiente, apresentando score de eficiência de 0,786, ou 78,6\%. Comparando-se os resultados desta pesquisa com os de Fonseca e Ferreira (2009) identifica-se uma maior ineficiência das microrregiões mineiras, uma vez que o menor score de eficiência das microrregiões de Minas Gerais foi 0,3181 , ou $31,81 \%$.

A partir do calculo do DEA pode-se identificar, além das DMUs eficientes e não eficientes, os benchmark daquelas não eficientes, ou seja, quais cidades as DMUs não eficientes deve ter por referência para, assim, alcançar sua eficiência plena (score de eficiência igual a 1, ou 100\%), no caso desta pesquisa os benchmark indicam quais microrregiões devem servir de exemplo àquelas não eficientes. A relação dos benchmarks das microrregiões catarinenses não eficientes é apresentada na tabela 4.

Tabela 4 - Benchmarks das microrregiões não eficientes de Santa Catarina

\begin{tabular}{l|c}
\hline \multicolumn{1}{c|}{ Microrregiões (DMUs) } & Benchmark \\
\hline Rio do Sul & Florianópolis e Tabuleiro \\
\hline Tubarão & Chapecó e Tabuleiro \\
\hline Itajaí & Blumenau, Chapecó, Concórdia e São Bento do Sul \\
\hline Criciúma & Tabuleiro e Xanxerê \\
\hline Joaçaba & Campos de Lages, Chapecó, Tabuleiro e Xanxerê \\
\hline Canoinhas & Araranguá, Chapecó e São Bento do Sul \\
\hline Joinville & Chapecó e Tabuleiro \\
\hline
\end{tabular}


Fonte: Dados da Pesquisa.

A fim de exemplificar a interpretação dos benchmarks analisa-se a microrregião de Joinville, visto que foi a menos eficientes dentre as microrregiões analisadas. Com base na tabela 4 a microrregião de Joinville deve orientar-se pelas microrregiões de Chapecó e Tabuleiro para obter melhor eficiência. Desta forma, para que o estado de Santa Catarina obtenha maior eficiência quanto ao uso dos recursos do Sistema Único de Saúde é oportuno às microrregiões não eficientes listadas na tabela 4 orientarem-se pelos seus respectivos benchmarks.

De modo geral a análise da eficiência do atendimento do SUS nas microrregiões catarinenses aponta que o estado de Santa Catarina é eficiente, podendo, entretanto, ainda obter maior eficiência. Baseando-se no referencial teórico abordado, pode-se inferir que o estado analisado, apesar das limitações de recursos já enfatizadas em estudos anteriores (MARINHO, 2003; MILLS; SPANCER, 2005; KATHARAKI, 2008) tem obtido resultados positivos quanto ao uso dos recursos disponíveis.

\section{CONCLUSÃO}

Este estudo objetivou avaliar a eficiência do atendimento do SUS das vinte microrregiões do estado de Santa Catarina através da análise envoltória de dados (DEA) utilizando dados de 2009 disponíveis no sítio oficial do DATASUS. Para consecução do objetivo primeiramente utilizou-se estatística descritiva, com o intuito de caracterizar a amostra da pesquisa. Num segundo momento efetuou-se o cálculo do score de eficiência das microrregiões catarinense através do DEA, utilizando o método BCC com orientação ao produto.

A partir do emprego do modelo DEA na construção da fronteira de eficiência e admitindo-se retornos variáveis com orientação ao produto, os resultados indicam significativa eficiência das microrregiões de Santa Catarina. Das 20 microrregiões analisadas, 13 apresentaram escores de eficiência iguais a 1, ou seja, $65 \%$ da amostra é eficiente.

Apesar da maioria das microrregiões se apresentarem como eficientes, há microrregiões não eficientes, como a de Joinville, considerada a menos eficiente do estado, cujo escore de eficiência de 0,786 , indicando que, para atingir a eficiência plena (escore igual a 1), sua produção deveria crescer 0,214 , mantendo-se os recursos disponíveis.

A eficiência média do estado de Santa Catarina, quanto ao atendimento do SUS, foi estimada em 0,9704, ou 97,04\%. Este resultado indica uma alta eficiência média do estado catarinense, bem como uma maior otimização dos recursos se comparada à eficiência do estado de Minas Gerais e quanto aos hospitais filantrópicos brasileiros. Se comparado ao estudo de Cesconetto, Lapa e Calvo (2008) percebe-se uma melhoria no que tange a eficiência do estado de Santa Catarina.

Por meio da análise foi possível identificar também as microrregiões não eficientes e, para estas, seus benchmarks, ou seja, as microrregiões pelas quais devem orientar-se a fim de obterem maior eficiência quanto ao uso de seus recursos destinados à saúde. Destaca-se, entretanto que, ainda que identificadas microrregiões não eficientes, o estado pode ser considerado eficiente, dado sua eficiência média ser de $97,04 \%$ e $75 \%$ da amostra apresentar escore de eficiência acima da média do estado.

Quanto à limitação dos recursos destaca pela literatura (MARINHO, 2003; MILLS; SPANCER, 2005; KATHARAKI, 2008) pode-se concluir que as microrregiões de Santa Catarina tem otimizado seus recursos tendo em vista atender a maior parcela de pacientes possíveis. Como limitação a esta pesquisa deve-se salientar que a literatura destaca que a limitação de recursos fronte a um grande número de atendimentos pode prejudicar a qualidade custos (MORRISEY; WEDIG; HASSAN, 1996; MILLS; SPENCER, 2005) e tal aspecto não 
foi analisado de modo que a eficiência apontada neste estudo dita-se apenas sobre a ociosidade de recursos e não aborda questões de qualidade.

Recomenda-se, para pesquisas futuras, que seja analisada a eficiência das microrregiões de Santa Catarina e, consequentemente, a do estado sob uma abordagem qualitativa com o objetivo de analisar a qualidade do atendimento do SUS para comparação da eficiência na utilização de recursos com a qualidade do serviço prestado. Uma segunda recomendação consiste na replicação da metodologia proposta neste estudo em outras amostras a fim de se obter um panorama mais abrangente quanto à eficiência do atendimento do SUS no Brasil bem como a comparabilidade dos resultados.

\section{REFERÊENIAS}

AL-SHAMMARI, M. A multi-criteria data envelopment analysis model for measuring the productive efficiency of hospitals. International Journal of Operations \& Production Management. v. 19, n. 9, p. 879-891, 1999.

BANKER, R. D.; CHARNES, A.; COOPER, W. W. Some Models for Estimating Technical and Scale Inefficiencies in Data Envelopment Analysis. Management Science. v. 30, n. 9, p. 1078-1092, 1984.

BARNUM, D. T.; WALTON, S. M.; SHIELDS, K. L.; SCHUMOCK, G. T. Measuring hospital efficiency with data envelopment analysis: Non-substitutable vs. substitutable inputs and outputs. Journal of Medical Systems. v. 35, n. 6, p. 1393-1401, 2011.

CESCONETTO, A.; LAPA, J. dos S.; CALVO, M. C. M. Avaliação da Eficiência produtiva de hospitais do SUS de Santa Catarina. Cadernos de Saúde Pública. v. 24, n. 10, p. 24072417, out. 2008 .

CHARNES, A.; COOPER, W. W.; RHODES, E. Measuring the Efficiency of Decision Making Units. European Journal of Operational Research. v. 2, n. 6, p. 429-444, 1978.

CHU, Y. The productive efficiency of Chinese hospitals. China Economic Review. v. 22, n. 3, p. 428-439, set. 2011.

CHUANG, C.; CHANG, P.; LIN, R. An Efficiency Data Envelopment Analysis Model Reinforced by Classification and Regression Tree for Hospital Performance Evaluation. Journal of Medical Systems. v. 35, n. 5, p. 1075-1083, 2011.

CUNHA, J. A. C. da. Avaliação de Desempenho e eficiência em organizações de Saúde: um estudo em hospitais filantrópicos. 2011. Tese (Doutorado em Controladoria e Contabilidade) - Faculdade de Economia, Administração e Contabilidade, Universidade de São Paulo, São Paulo, 2011.

DENEFFE, D.; MASSON, R. What do not-for-profit hospitals maximize? International Journal of Industrial Organization. v. 20, n. 4, p. 461-492, 2002.

FERLIE, E.; ASBURNER, L.; FITZGERALD, L.; PETTIGREW, A. A nova Administração pública em ação. Brasília: UNB/ENAP, 1999. 
FERREIRA, C. M. de C.; GOMES, A. P. Introdução à análise envoltória de dados: modelos e aplicações. Minas Gerais: Editora UFV, 2009.

FONSECA, P. C.; FERREIRA, M. A. M. Investigação dos Níveis de Eficiência na utilização de Recursos no setor de saúde: uma análise das microrregiões de Minas Gerais. Saúde e Sociedade. v. 18, n. 2, p.199-213, 2009.

GOMES, E. G.; MANGABEIRA, J. A. C. Uso de Análise Envoltória de Dados em Agricultura: o caso de Holambra. Engevista. v. 6, n. 1, p. 19-27, abr. 2004.

GONÇALVEZ, L. Análise da eficiência dos hospitais SA e SPA segundo uma abordagem de fronteira de eficiência. 2008. Tese (Doutorado em Gestão) - Instituto Universitário de Lisboa, Lisboa, 2008.

GUERRA, M.; SOUZA, A. A. de.; MOREIRA, D. R. Performance Analysis: A study using data envelopment analysis in 26 Brazilian Hospitals. Journal of Health Finance. v. 38, n. 4, p. 19-35, 2012.

IBAÑEZ, N.; VECINA NETO, G. Modelos de gestão e o SUS. Ciência \& Saúde Coletiva. Rio de Janeiro, v. 12, p. 1831-1840, 2007.

JUNQUEIRA, L. A. P. Gerência dos serviços de saúde. Cadernos de Saúde Pública. v. 6, n. 3, p. 247-259, jul./set. 1990.

KATHARAKI, M. Approaching the management of hospital units with an operation research technique: the case of 32 Greek obstetric and gynaecology public units. Health Policy. v. 85, n. 1, p. 19-31, jan. 2008.

KEELER, E.; MELNICK, G.; ZWANZIGER, J. The changing effects of competition on nonprofit and for-profit hospital pricing behavior. Journal of Health Economics. v. 18, p. 69-86, 1999.

MACEDO, M. A. S.; BARBOSA, A. C. T. C.; CAVALCANTE, G. T. C. Desempenho de agências bancárias no Brasil: aplicando análise envoltória de dados (DEA) a indicadores relacionados às perspectivas do BSC. Revista Economia \& Gestão. v. 9, n. 19, p. 65-84, jan./abr. 2009.

MARINHO, A. Avaliação da eficiência técnica nos serviços de saúde nos municípios do estado do Rio de Janeiro. Revista Brasileira de Economia. v. 57, n. 2, p. 515-534, jul./set. 2003.

MILLS, A. E.; SPENCER, E. M. Values based decision making: a tool for achieving the goals of healthcare. HEC Forum. v. 17, n. 1, p. 18-32, 2005.

MORRISEY, M. A.; WEDIG, G. J.; HASSAN, M. Do nonprofit hospitals pay their way? Health Affairs. v. 15, n. 4, p. 132-144, 1996.

NICOLA, A. de.; GITTO, S.; MANCUSO, P. Uncover the predictive structure of healthcare efficiency applying a bootstrapped data envelopment analysis. Expert Systems with Applications. v. 39, n. 12, p. 10495-10499, set. 2012. 
PAULY, M. V. Nonprofit firms in medical markets. American Economic Review. v. 77, n. 2, p. 2757-262, maio, 1987.

PEIRÓ, S.; ARTELLS, J. J.; MENEU, R. Identificación y priorización de actuaciones de mejora de la eficiência en el Sistema Nacional de Salud. Gaceta Sanitaria. v. 25, p. 95-105, 2011.

POLITELO, Leandro; KAVESKI, Itzhak Simão David; GODOY, Nádia Nara de; CUNHA, Paulo Roberto. Níveis de eficiência na utilização de recursos no setor de saúde: uma análise na mesorregião do Vale do Itajaí - Santa Catarina. In: ENCONTRO DA DIVISÃO DE ADMINISTRAÇÃO PÚBLICA, 5., 2012, Salvador. Anais... Rio de Janeiro: EnAPG, 2012.

REDER, M. W. Some problems in the economics of hospitals. The American Economic Review, v. 55, n. 1/2, p. 472-480, mar. 1965.

RICHARDSON, R. J. Pesquisa social: métodos e técnicas. 3. ed. São Paulo: Atlas, 1999.

SANTOS, C. M. dos.; CARVALHO, H. R.; LÍRIO, V. S. Eficiência do atendimento do SUS no estado de Minas Gerais. In: Seminário sobre a Economia Mineira, 8, 2008. Anais... Belo Horizonte: UFMG/Cedeplar, 2008.

SARKIS, J.; TALLURI, S. International Journal of Operation \& Production Management. v. 22, n. 3, p. 306-313, 2002.

SILVA, A. M. M. da; BRANDALIZE, A. A moderna administração hospitalar. Terra e Cultura. v. 22, n. 42, p. 56-67, jan./jun. 2006.

SOUZA, I. V.; NISHIJIMA, M.; ROCHA, F. Eficiência do setor hospitalar nos municípios paulistas. Economia Aplicada. v. 14, n. 1, p.51-66, 2010.

STAAT, M. Efficiency of hospitals in Germany: a DEA-bootstrap approach. Applied Economics. v. 38, p. 2255-2263, 2006.

VERGARA, S. C. Projetos e relatórios de pesquisa em administração. 3. ed. São Paulo: Atlas, 2000.

VIACAVA, F.; UGÁ, M. A. D.; PORTO, S.; LAGUARDIA, J.; MOREIRA, R. da S. Avaliação de Desempenho de Sistemas de Saúde: um modelo de análise. Ciência \& Saúde Coletiva. v. 17, n. 4, p. 921-934, abr. 2012. 